\title{
A systematic review of the impact of antifungal stewardship interventions in the United States
}

\author{
Emily Hart, Melanie Nguyen, Meghan Allen, Collin M. Clark and David M. Jacobs*
}

\begin{abstract}
Background: Antimicrobial resistance is a widely recognized public health threat, and stewardship interventions to combat this problem are well described. Less is known about antifungal stewardship (AFS) initiatives and their influence within the United States. The purpose of this study was to evaluate evidence on the impact of AFS interventions on clinical and performance measures.

Methods: A systematic review of English language studies identified in the PubMed and EMBASE databases was performed through November 2017. The review was conducted in accordance with PRISMA. Search terms included antifungal stewardship, antimicrobial stewardship, Candida, candidemia, candiduria, and invasive fungal disease. Eligible studies were those that described an AFS program or intervention occurring in the US and evaluated clinical or performance measures.

Results: Fifty-four articles were identified and 13 were included. Five studies evaluated AFS interventions and reported clinical outcomes (mortality and length of stay) and performance measures (appropriate antifungal choice and time to therapy). The remaining eight studies evaluated general stewardship interventions and reported data on antifungal consumption. All studies were single center, quasi-experimental with varying interventions across studies. AFS programs had no impact on mortality (3 of 3 studies), with an overall rate of $27 \%$ in the intervention group and $23 \%$ in the non-intervention group. Length of stay (5 of 5) was also similar between groups (range, 9-25 vs. 11-22). Time to antifungal therapy improved in 2 of 5 studies, and appropriate choice of antifungal increased in 2 of 2 studies. Antifungal consumption was significantly blunted or reduced following stewardship initiation (8 of 8), although a direct comparison between studies was not possible due to a lack of common units.
\end{abstract}

Conclusion: The available evidence suggests that AFS interventions can improve performance measures and decrease antifungal consumption. Although this review did not detect improvements in clinical outcomes, significant adverse outcomes were not reported.

Keywords: Antifungal stewardship, Antimicrobial stewardship, Invasive fungal disease, Antifungal agents

\section{Introduction}

Antimicrobial resistance is a growing public health challenge that poses a global threat [1]. In the United States, at least 2 million people acquire and at least 23,000 people die each year from an antibiotic-resistant infection [2]. Approaches to optimize antibiotic use

\footnotetext{
*Correspondence: dmjacobs@buffalo.edu
} Department of Pharmacy Practice, School of Pharmacy and Pharmaceutical Sciences, University at Buffalo, 316 Pharmacy Building, Buffalo, NY, USA and contain antimicrobial resistance have been recommended to preserve the benefits of antibiotics and provide the best patient care. Antimicrobial stewardship programs (ASPs) have received particular attention because of their focus on improving health outcomes and patient care. The Infectious Diseases Society of America (IDSA) and the Society for Healthcare Epidemiology of America published an updated guideline on the implementation of ASPs within inpatient populations [3]. ASPs are defined as coordinated efforts 
designed to improve the appropriate use of antibiotics by promoting the selection of the optimal antibiotic regimen [3]. The benefits of ASPs are well documented and include improved patient outcomes, reduced Clostridium difficile infections, and optimized resource utilization across the continuum of care [3-8].

Although ASPs have primarily focused on antibiotics, antifungal resistance is a growing and emerging threat [9]. Candida infections due to fluconazole- and echinocandin-resistant strains are increasingly prevalent and comprise over $70 \%$ of resistant isolates from Candida glabrata or Candida krusei species [10, 11]. Candida auris is also an emerging multi-drug resistant pathogen with cases or outbreaks reported in over 20 countries since its first discovery in 2009 [12]. This is especially concerning given that $C$. auris isolates can be resistant to all three of the main classes of antifungal drugs, and cases can go undetected as it is commonly misidentified in clinical laboratories [12]. Further, although antifungal resistance is common in Candida species, emerging threats also include azole-resistant Aspergillus fumigatus [13]. Several studies have shown that antifungal agent use can deviate from guidelines and that this has a negative impact on patient outcomes [14-17]. Appropriate antifungal use is an important factor in fighting drug resistance [18]. Given the rise in drug resistance and the documented inappropriate use of antifungals, the implementation of formal antifungal stewardship (AFS) programs is becoming increasingly important.

Antifungal stewardship inherently has different complexities and clinical priorities to antimicrobial stewardship, but ultimately they share a common goal of improving appropriate drug use through regimen optimization [18-20]. AFS programs are emerging as a sub-specialty of ASPs, yet the literature on these programs is sparse. The purpose of this systematic review is to summarize evidence on AFS programs in the United States and evaluate their impact on clinical and performance outcomes. We focus on US-based programs given the differences in healthcare systems and resources around the world (i.e., settings where antifungals are available without a prescription).

\section{Methods}

\section{Search strategy}

A comprehensive search for articles relevant to ASP implementation, with a focus on programs specifically obtaining data on AFS, was conducted through November 2017. Two researchers independently conducted the search, and inclusion was determined based on a consensus of the relevance of the identified study. The literature search was carried out through the PubMed and EMBASE online databases utilizing the following terms: antifungal AND stewardship, antifungal stewardship, antimicrobial stewardship AND candida AND invasive fungal, antimicrobial stewardship AND invasive fungal, antimicrobial stewardship AND candida, antimicrobial stewardship AND candiduria AND invasive fungal, invasive fungal disease AND stewardship, stewardship AND candida AND candidemia AND candiduria, stewardship AND candida, stewardship AND candidemia, stewardship AND candiduria. In addition, to increase completeness the reference lists of relevant articles were searched.

\section{Inclusion and exclusion criteria}

Studies were included if: (a) the article described an AFS program or intervention; (b) the program was implemented in the United States; and (c) the article reported an AFS program containing data on clinical or performance measures. Exclusion criteria were determined according to the following components: (a) not written in English; (b) study did not include an intervention; and (c) did not evaluate an outcome of interest. Outcomes of interest were divided into performance measures (appropriate fungal choice, time to therapy, and antifungal consumption) and clinical measures (mortality and length of stay).

\section{Study selection and data extraction}

Both researchers independently screened all titles and abstracts identified in the literature search. All abstracts were considered if they met the inclusion criteria, and full-text articles were then retrieved for further review. All disagreements over eligibility were resolved via discussion between the researchers.

After obtaining the full-text articles meeting the inclusion criteria, two investigators extracted data using a standardized form that included study title, year of publication, author, objectives, design, patient population, duration, site, intervention description, and findings pertaining to outcomes of interest. An Excel spreadsheet (Microsoft Corp., Redmond, WA) compiling all variables to be extracted was used to ensure data extraction reproducibility and completeness. A second researcher further reviewed the extracted data to verify the necessity for the data. Any disagreements on data inclusion were confirmed through discussion between all the researchers.

\section{Synthesis of results}

The Preferred Reporting Items for Systematic Reviews and Meta-Analyses (PRISMA) checklist was used to guide the systematic review [21]. Due to variability in interventions, patient populations, and outcome measures, extracted data were summarized descriptively. Conclusions were drawn based on qualitative synthesis of the findings. 


\section{Results}

\section{Search results}

A total of 2083 studies were initially screened for inclusion by title and abstract. After excluding duplicates, non-relevant studies, non-interventional studies, and studies performed outside the US, 54 articles were eligible for full-text assessment. Of these, 41 did not report data on clinical and performance measures and were excluded. Thus, 13 articles were included in the systematic review (Fig. 1).

\section{Study characteristics}

Of the included articles, five evaluated AFS interventions and reported clinical outcomes (mortality and length of stay) and performance outcomes (appropriate antifungal choice and time to therapy) [22-26]. These studies are summarized in Table 1 . The remaining eight studies, summarized in Table 2, evaluated general antimicrobial stewardship interventions and reported data on antifungal consumption [27-34].
All studies were single-centered and quasi experimental in design with the earliest publication in 2001. Any data that did not pertain to outcomes of interest or antifungal agents were not included in the review. The fives studies that included clinical and performance outcomes had a study duration of 1-2 years and recorded data on 411 patients, the majority with a diagnosis of Candida infections. The eight studies used to reference antifungal consumption had an average of 4 years study duration, where each had a statistically significant decrease in antifungal use.

\section{Interventions}

Intervention type and implementation varied across studies (Table 1). The five studies that evaluated AFS interventions included: implementation of a care bundle (1 study) [22], AFS pharmacist recommendations (1 study) [23], and development of a diagnostic tool (3 studies) [24-26]. Of the eight studies that evaluated general antimicrobial stewardship, many programs implemented multiple

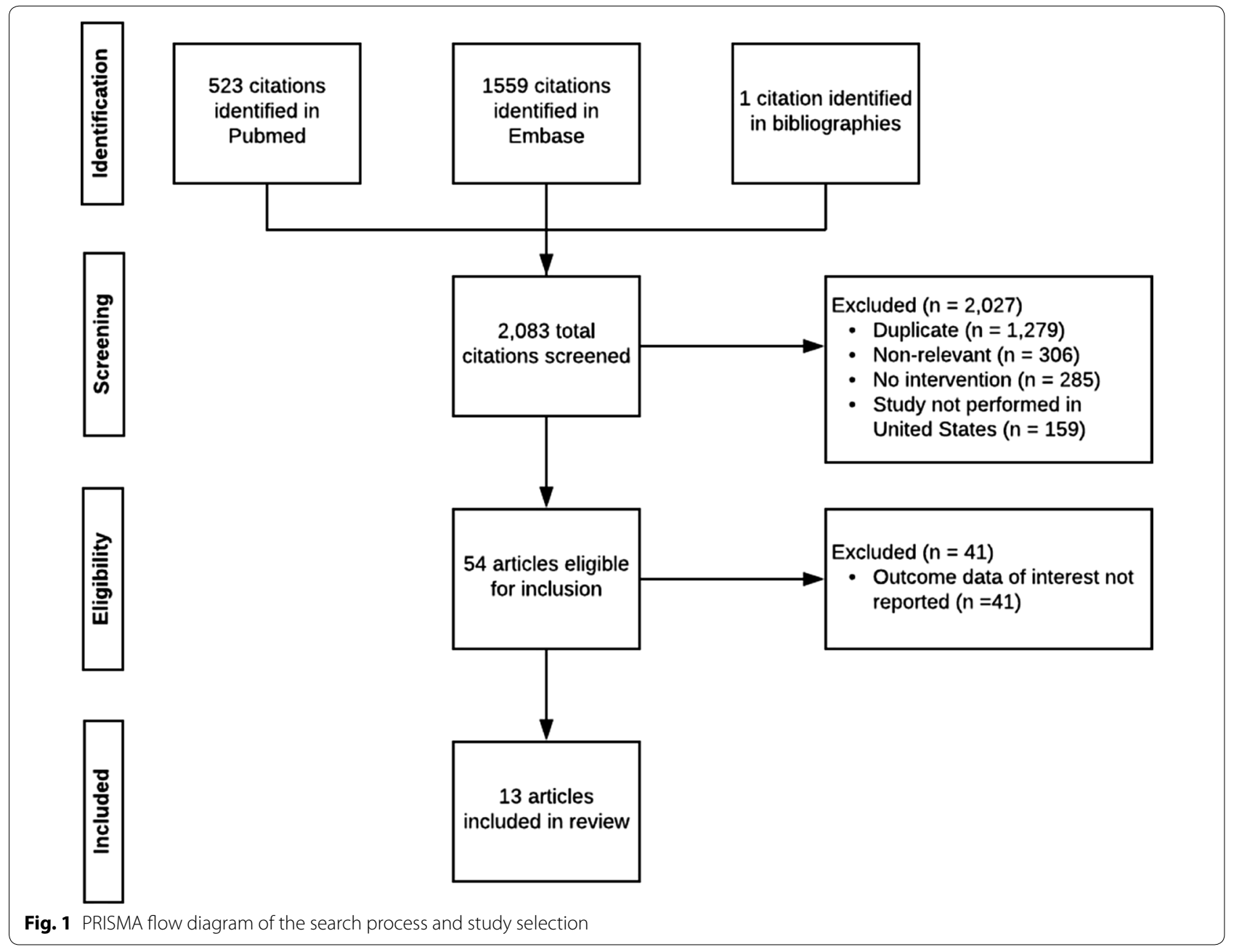




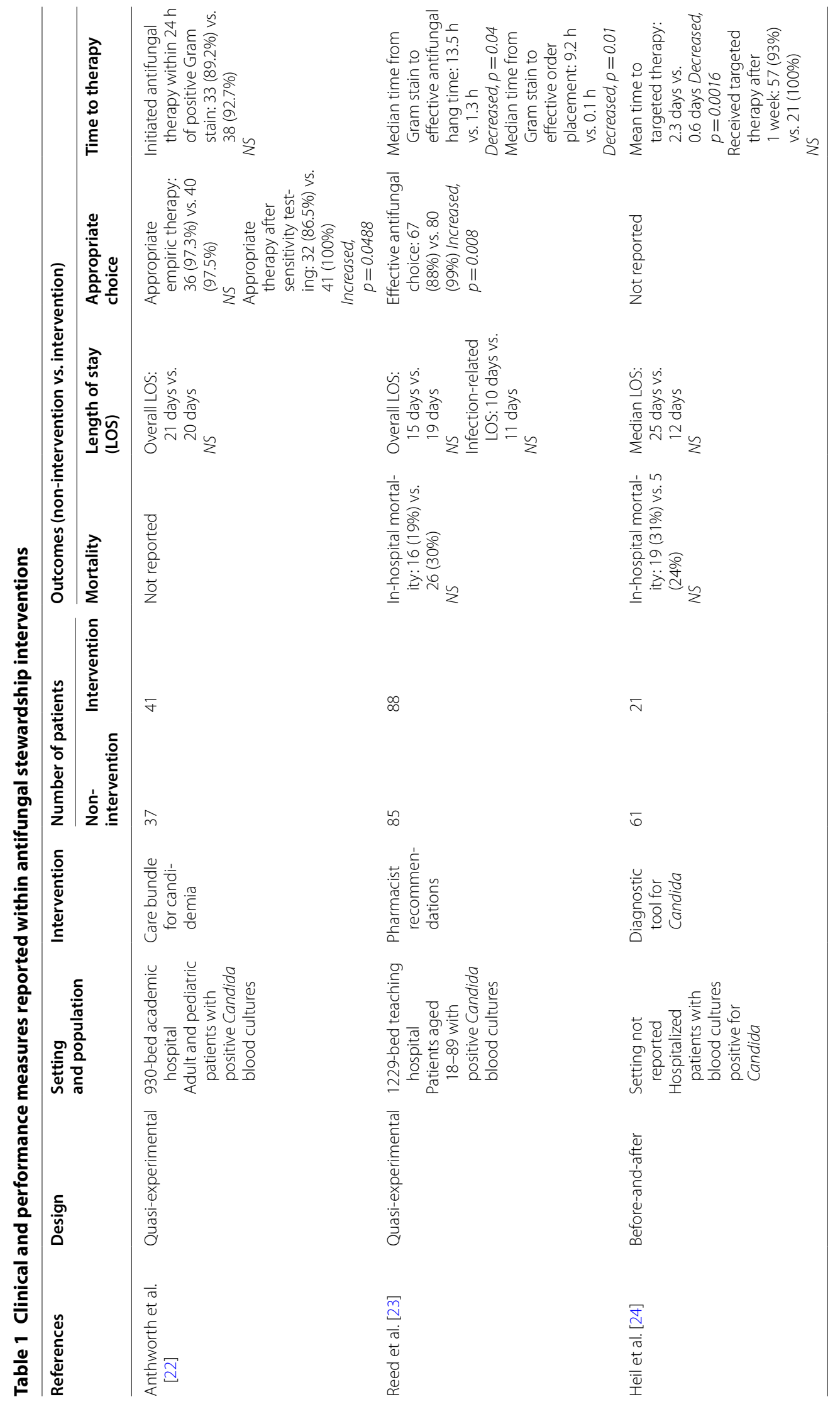




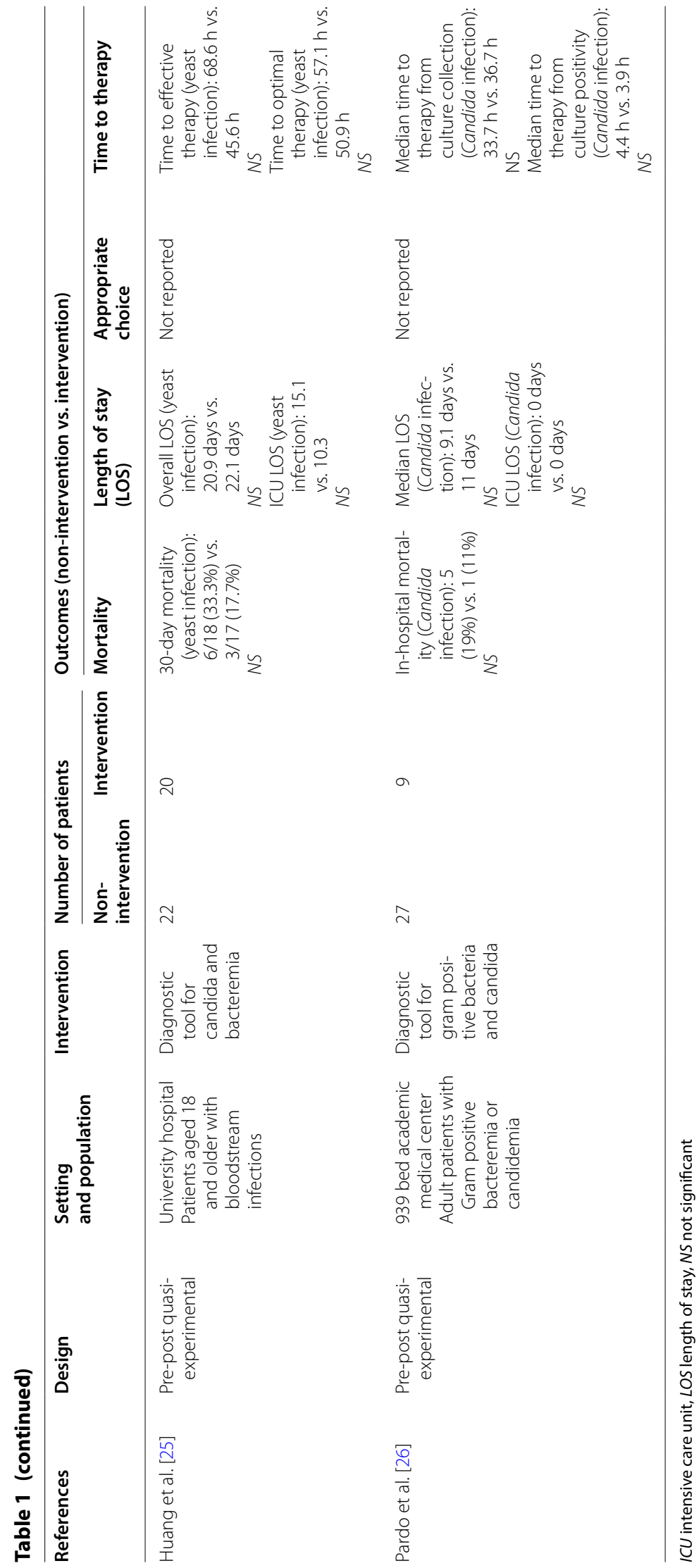




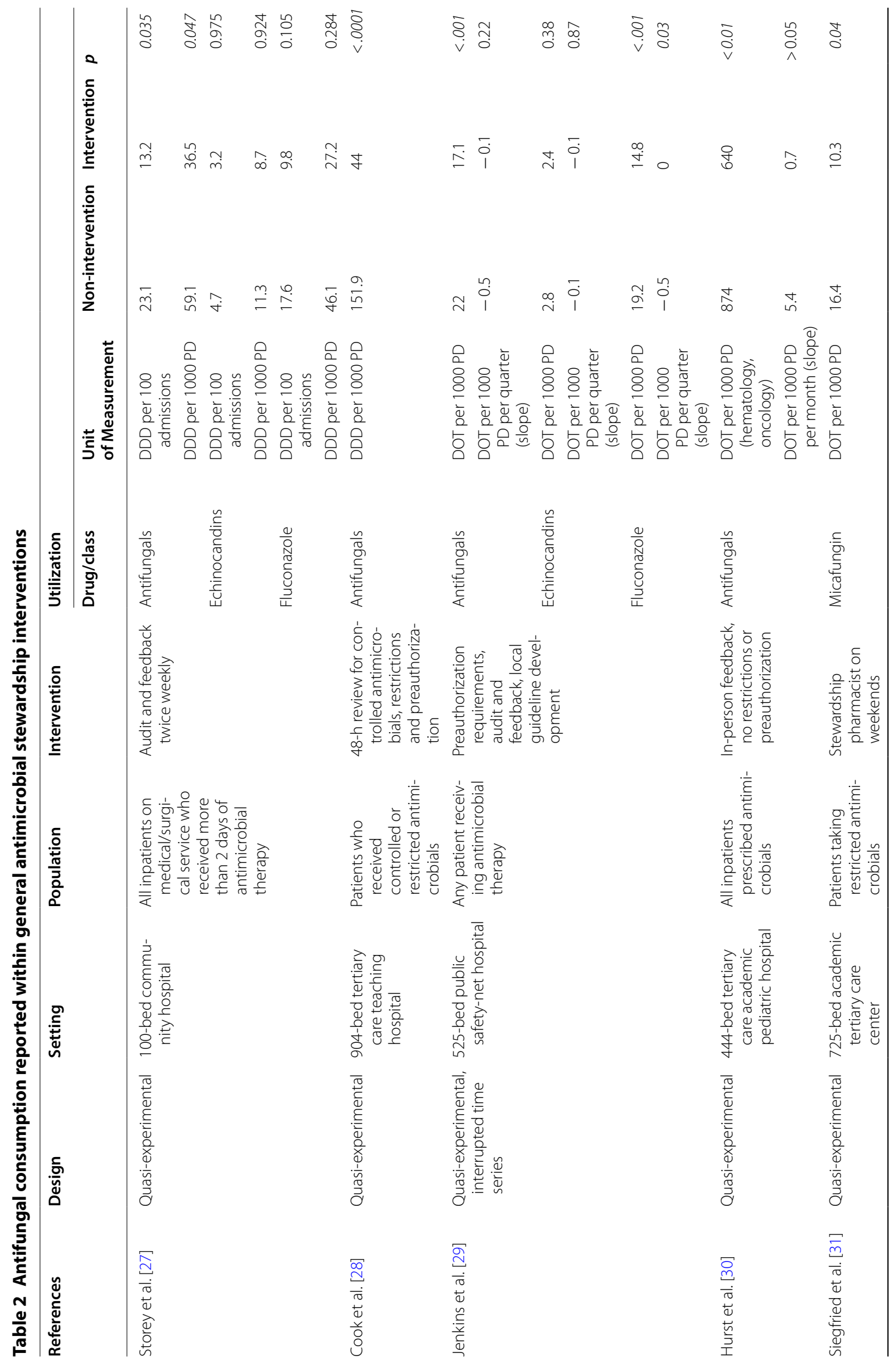




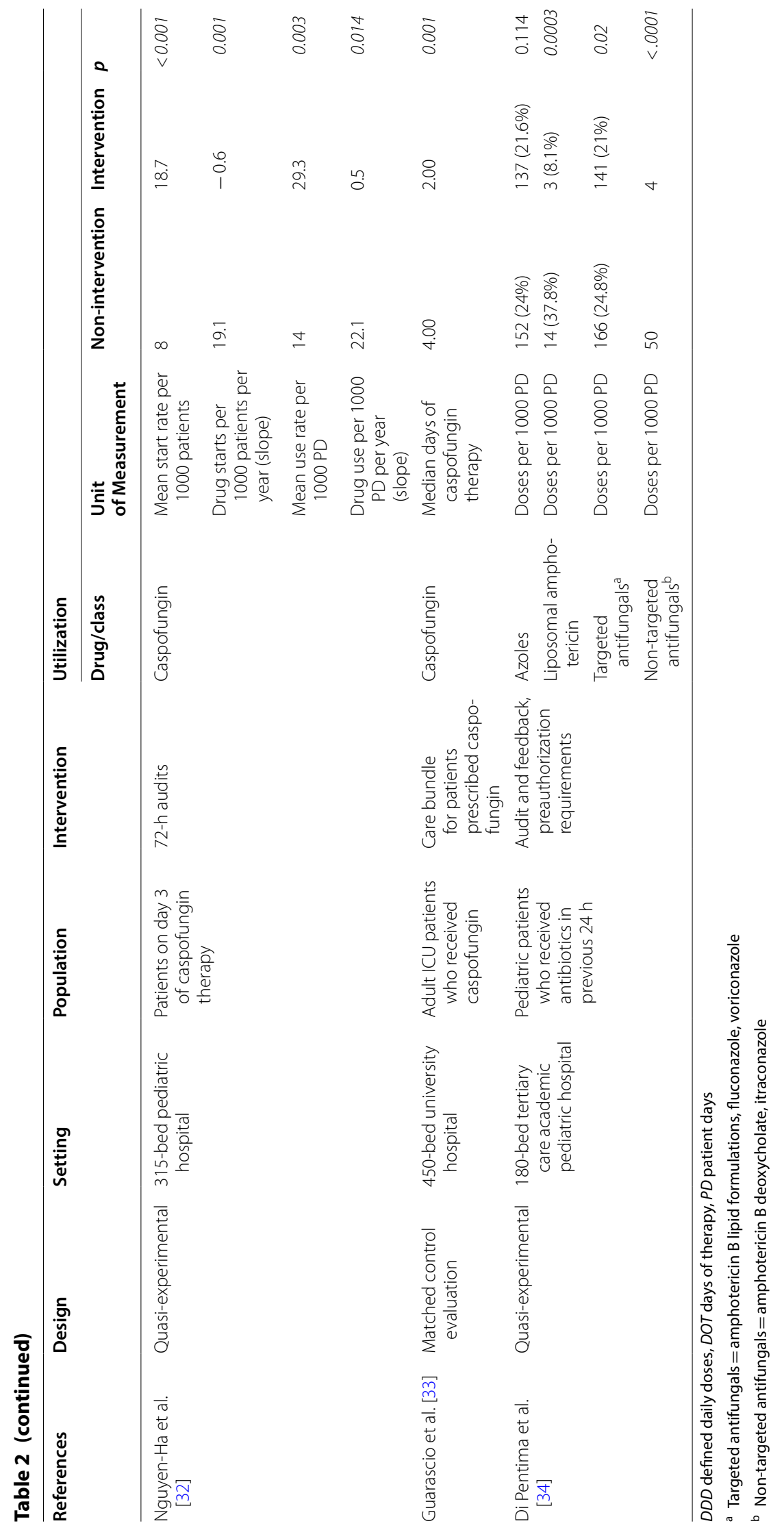


interventions. These included audit and feedback (5 studies) $[27,29,30,32,34]$, preauthorization requirements or restriction (3 studies) [28, 29, 34], local guideline development (1 study) [29], care bundle development (1 study) [33], in-person feedback with no restrictions or preauthorization requirements (1 study) [30], and pharmacist stewardship coverage on weekends (1 study) [31].

\section{Performance outcomes}

\section{Appropriate antifungal choice}

Two studies evaluated appropriate choice of antifungal $[22,23]$. A higher percentage of patients were given the appropriate choice of antifungal in both studies. In one study, appropriate therapy after sensitivity testing was significantly higher in intervention vs. non-intervention groups, though rate of appropriate empiric therapy was unchanged. In the other study, rate of effective choice of antifungal was increased in the intervention vs. nonintervention group.

\section{Time to therapy}

Evaluation of time to antifungal therapy varied across five studies (Table 1). Four studies reported mean or median time to therapy in hours [23-26], and two studies reported percentages of patients receiving therapy within a specified timeframe [22, 24]. Time to therapy was improved in two of five studies. In one study, median time from Gram stain to effective antifungal hang time and order placement was significantly decreased, and in one study mean time to targeted therapy was decreased. The percentage of patients who received therapy within a timeframe was unchanged in two of two studies.

\section{Antifungal consumption}

Of the eight studies that evaluated general antimicrobial stewardship interventions, all reported data on antifungal consumption (Table 2). Various units were used to describe antifungal consumption including defined daily doses per 1000 patient-days or per 100 admissions (2 studies) [27, 28], days of therapy per 1000 patientdays (3 studies) [29-31], mean drug start and use rates (1 study) [32], median days of therapy (1 study) [33], and doses per 1000 patient-days (1 study) [34]. Due to the lack of common units, a direct quantitative comparison between studies was not possible. However, all studies reported either a significant decrease in use (7 of 8) or blunting of upward trend in use (1 of 8) of antifungal agents. Five studies evaluated all antifungal agents, and consumption was decreased in all five studies. Two studies evaluated echinocandins as a class, and consumption was unchanged in both studies. Caspofungin use was decreased in two studies, and micafungin use was decreased in one of one study. Consumption of azoles as a class was unchanged in one of one study, and fluconazole use was significantly decreased in one of two studies. Liposomal amphotericin B utilization was significantly decreased in one of one study.

\section{Clinical outcomes \\ Mortality}

Four of five studies that evaluated AFS interventions reported data on mortality outcomes (Table 1) [22-26]. Of these, three reported in-hospital mortality and one reported 30-day mortality. All four studies found no significant change in mortality between intervention vs. non-intervention groups. In-hospital mortality occurred at a rate of $27 \%(32 / 118)$ in intervention groups and $23 \%$ (40/173) in non-intervention groups across three studies. In one study, 30-day mortality was $18 \%(3 / 17)$ in the intervention group and 33\% (6/18) in the non-intervention group.

\section{Hospital length of stay}

All five studies that evaluated AFS interventions reported hospital length of stay (LOS) outcomes (Table 1). Overall LOS was unchanged across five of five studies and ranged from 9 to 25 days in intervention groups vs. 11-22 days in non-intervention groups. Intensive care unit LOS was also unchanged across two of five studies (range $0-10 \mathrm{vs.}$ $0-15$ days) $[25,26]$. One study reported infection-related LOS (11 vs. 10 days), which was not significantly changed by the intervention [23].

\section{Discussion}

Antifungal stewardship is an important component of a stewardship program given the rise in antifungal resistance and associated poor clinical outcomes $[9,18]$. This is especially pertinent given the recent recognition and challenges associated with multi-drug resistant $C$. auris [12]. In order to properly combat antifungal resistance, additional AFS strategies and programs will be necessary.

The stewardship interventions varied across studies, but common stewardship interventions were used including audit and feedback or preauthorization requirements $[27-29,34]$. Three studies were based on the introduction of a diagnostic tool for Candida species [24-26]. Similar to the IDSA recommendations for ASPs, the core members of an AFS team should include an infectious diseases specialist, clinical pharmacist, and a clinical microbiologist $[3,19]$. Pharmacist recommendations were used as the primary intervention in two studies, which has been shown to improve care and clinical outcomes within ASPs [23, 31, 35]. Within the included AFS studies, all five articles reported including an infectious diseases-trained physician and a pharmacist as core members of the AFS programs. Microbiology was 
included in all studies, although it was unclear whether a clinical microbiologist was one of the core members of the AFS program. The formation of a multidisciplinary team with the necessary expertise will be key to the development and success of any AFS program [19].

Antifungal consumption was the most common outcome measure reported. Various approaches were used to describe consumption including defined daily doses, days of therapy, and dose adjusted to hospital bed occupancy. The use of antifungal days of therapy is the preferred metric according to IDSA guidelines as it is not impacted by dose adjustments and can be used in pediatrics where dosing is based on patient weight [3]. Notwithstanding the antifungal consumption metric, all studies reported decreased in antifungal use or blunting a previous upward trend in utilization. These decreases were apparent in studies reporting both overall antifungal utilization and those focusing on specific antifungal classes or drugs. Although it is clear that AFS can have a positive impact on antifungal consumption, the prescribing quality within these studies is not as clear. Only two studies evaluated whether appropriate antifungal therapy was prescribed. Both studies reported a higher percentage of patients given appropriate choices of antifungal therapy following implementation of an AFS intervention. The majority of studies did not evaluate appropriateness of antifungal prescribing as a process outcome. Previous research has shown a high proportion of inappropriate antifungal agent use including inadequate dosages or indications $[14,15,36]$. Given the overtreatment with antifungal therapy coupled with the rise in resistance, there should be greater focus on compliance with guideline recommendations as a reported performance measure.

Establishing the impact of AFS interventions on clinical outcomes should be a primary focus along with reporting antifungal utilization and other process outcomes. Only a few studies evaluated clinical outcomes including inhospital or 30-day mortality and overall hospital length of stay. Notably, no significant change was reported in these clinical outcomes following the implementation of an AFS program. A meta-analysis of the implementation of hospital-based ASPs also found no difference in mortality following program implementation [37]. ASPs were associated with a significant decrease in hospital length of stay; however, these findings were based on only four studies [37]. At the very least, our findings support previous reports that stewardship programs do not adversely affect the level of patient care by focusing antifungal therapy on patients who really need it. However, similar to antimicrobial stewardship, AFS programs will need to evaluate clinical outcomes and show improvements in care in order to justify additional resources beyond the cost savings associated with decreased antifungal consumption.

Our study should be interpreted in view of certain limitations. The major one is the scarcity of literature and evidence to support AFS programs. Studies focusing on AFS programs were primarily published after 2010, which is consistent with the emergence of this concept [38]. Another important limitation is that all included studies were non-randomized and were primarily single center, quasi-experimental designs. Further, specific conclusions were drawn from studies with small numbers of patients. In addition, we focused our search on studies within the United States, which may limit the generalizability of our findings. Given the differences in healthcare around the world, our focus was to better understand the impact of AFS programs within the US healthcare system.

\section{Conclusion}

Even though there is limited evidence on AFS programs and the interventions are highly variable, the evidence suggests that AFS effectively improves performance measures and decreases antifungal consumption. As an emerging field, AFS is similar to established ASPs, yet with different clinical priorities. Central to AFS expansion will be a standardized approach for the inclusion of core members within an AFS multidisciplinary team as well as comprehensively evaluating the quantity and quality of antifungal prescribing. Long-term evaluation is necessary to show the effect of AFS on patient and economic outcomes including mortality. Furthermore, as additional AFS studies become available, the development of guidelines will be necessary to benchmark best practices.

\section{Acknowledgements \\ Not applicable.}

\section{Authors' contributions}

DJ conceptualized and designed the study. $\mathrm{EH}, \mathrm{MN}, \mathrm{MA}$ collected and analyzed the data. EH, MA, CC, DJ interpreted the analysis. EH, MA, CC, DJ drafted the manuscript. CC and DJ critically revised the manuscript. All authors read and approved the final manuscript.

\section{Funding \\ None.}

Availability of data and materials

All relevant data included within the paper were obtained from articles available in Pubmed and EMBASE using the search strategy described in the paper.

Ethics approval and consent to participate

Not applicable.

Consent for publication

Not applicable.

Competing interests

The authors declare that they have no competing interests. 
Received: 24 May 2019 Accepted: 14 August 2019

Published online: 21 August 2019

\section{References}

1. Centers for Disease Control and Prevention. Antibiotic/antimicrobial resistance. https://www.cdc.gov/drugresistance/index.html. Accessed Apr 232019.

2. Centers for Disease Control and Prevention. Antibiotic resistance threats in the United States, 2013. https://www.cdc.gov/drugresistance/threa t-report-2013/pdf/ar-threats-2013-508.pdf. Accessed Apr 282019.

3. Barlam TF, Cosgrove SE, Abbo LM, MacDougall C, Schuetz AN, Septimus EJ, et al. Implementing an Antibiotic Stewardship Program: guidelines by the Infectious Diseases Society of America and the Society for Healthcare Epidemiology of America. Clin Infect Dis. 2016;62(10):e51-77.

4. Bishop J, Parry MF, Hall T. Decreasing Clostridium difficile infections in surgery: impact of a practice bundle incorporating a resident rounding protocol. Conn Med. 2013;77(2):69-75.

5. Valiquette L, Cossette B, Garant MP, Diab H, Pepin J. Impact of a reduction in the use of high-risk antibiotics on the course of an epidemic of Clostridium difficile-associated disease caused by the hypervirulent NAP1/027 strain. Clin Infect Dis. 2007;45(Suppl 2):S112-21.

6. Ashiru-Oredope D, Sharland M, Charani E, McNulty C, Cooke J, Group AAS. Improving the quality of antibiotic prescribing in the NHS by developing a new antimicrobial stewardship programme: start smart-then focus. J Antimicrob Chemother. 2012;67(Suppl 1):i51-63.

7. File TM Jr, Solomkin JS, Cosgrove SE. Strategies for improving antimicrobial use and the role of antimicrobial stewardship programs. Clin Infect Dis. 2011;53(Suppl 1):S15-22.

8. Fishman N. Antimicrobial stewardship. Am J Med. 2006;119(6 Suppl 1):S53-61 (discussion S2-S70).

9. Centers for Disease Control and Prevention. Antifungal resistance. 2019. https://www.cdc.gov/fungal/antifungal-resistance.html\#three. Accessed April 28, 2019

10. Vallabhaneni S, Cleveland AA, Farley MM, Harrison LH, Schaffner W, Beldavs ZG, et al. Epidemiology and risk factors for echinocandin nonsusceptible Candida glabrata bloodstream Infections: data from a Large Multisite Population-Based Candidemia Surveillance Program, 2008-2014. Open Forum Infect Dis. 2015;2(4):ofv163.

11. Lockhart SR, Etienne KA, Vallabhaneni S, Farooqi J, Chowdhary A, Govender NP, et al. Simultaneous emergence of multidrug-resistant Candida auris on 3 continents confirmed by whole-genome sequencing and epidemiological analyses. Clin Infect Dis. 2017;64(2):134-40.

12. Forsberg K, Woodworth K, Walters M, Berkow EL, Jackson B, Chiller T, et al. Candida auris: the recent emergence of a multidrug-resistant fungal pathogen. Med Mycol. 2019;57(1):1-12.

13. Verweij PE, Chowdhary A, Melchers WJ, Meis JF. Azole resistance in Aspergillus fumigatus: can we retain the clinical use of mold-active antifungal azoles? Clin Infect Dis. 2016;62(3):362-8.

14. Nivoix Y, Launoy A, Lutun P, Moulin JC, Phai Pang KA, Fornecker LM, et al. Adherence to recommendations for the use of antifungal agents in a tertiary care hospital. J Antimicrob Chemother. 2012;67(10):2506-13.

15. Jacobs DM, Dilworth TJ, Beyda ND, Casapao AM, Bowers DR. Overtreatment of asymptomatic candiduria among hospitalized patients: a multi-institutional study. Antimicrob Agents Chemother. 2018;62(1):e01464-517.

16. Munoz P, Rojas L, Cervera C, Garrido G, Farinas MC, Valerio M, et al. Poor compliance with antifungal drug use guidelines by transplant physicians: a framework for educational guidelines and an international consensus on patient safety. Clin Transplant. 2012;26(1):87-96.

17. Singh N, Wagener MM, Cacciarelli TV, Levitsky J. Antifungal management practices in liver transplant recipients. Am J Transplant. 2008;8(2):426-31.

18. Munoz P, Bouza E, group Cs. The current treatment landscape: the need for antifungal stewardship programmes. J Antimicrob Chemother. 2016;71 (suppl 2):ii5-12.

19. Agrawal S, Barnes R, Bruggemann RJ, Rautemaa-Richardson R, Warris A. The role of the multidisciplinary team in antifungal stewardship. J Antimicrob Chemother. 2016;71(Suppl 2):ii37-42.
20. Aguado JM, Silva JT, Bouza E. Conclusion and future perspectives on antifungal stewardship. J Antimicrob Chemother. 2016;71(suppl 2):ii43-4.

21. PRISMA Checklist. http://prisma-statement.org/PRISMAStatement/Check list.aspx.

22. Antworth A, Collins CD, Kunapuli A, Klein K, Carver P, Gandhi T, et al. Impact of an antimicrobial stewardship program comprehensive care bundle on management of candidemia. Pharmacotherapy. 2013;33(2):137-43.

23. Reed EE, West JE, Keating EA, Pancholi P, Balada-Llasat JM, Mangino JE, et al. Improving the management of candidemia through antimicrobial stewardship interventions. Diagn Microbiol Infect Dis. 2014;78(2):157-61.

24. Heil EL, Daniels LM, Long DM, Rodino KG, Weber DJ, Miller MB. Impact of a rapid peptide nucleic acid fluorescence in situ hybridization assay on treatment of Candida infections. Am J Health Syst Pharm. 2012:69(21):1910-4

25. Huang AM, Newton D, Kunapuli A, Gandhi TN, Washer LL, Isip J, et al. Impact of rapid organism identification via matrix-assisted laser desorption/ionization time-of-flight combined with antimicrobial stewardship team intervention in adult patients with bacteremia and candidemia. Clin Infect Dis. 2013;57(9):1237-45

26. Pardo J, Klinker KP, Borgert SJ, Butler BM, Giglio PG, Rand KH. Clinical and economic impact of antimicrobial stewardship interventions with the FilmArray blood culture identification panel. Diagn Microbiol Infect Dis. 2016;84(2):159-64.

27. Storey DF, Pate PG, Nguyen AT, Chang F. Implementation of an antimicrobial stewardship program on the medical-surgical service of a 100-bed community hospital. Antimicrob Resist Infect Control. 2012;1 (1):32.

28. Cook PP, Gooch M. Long-term effects of an antimicrobial stewardship programme at a tertiary-care teaching hospital. Int J Antimicrob Agents. 2015;45(3):262-7.

29. Jenkins TC, Knepper BC, Shihadeh K, Haas MK, Sabel AL, Steele AW, et al. Long-term outcomes of an antimicrobial stewardship program implemented in a hospital with low baseline antibiotic use. Infect Control Hosp Epidemiol. 2015;36(6):664-72.

30. Hurst AL, Child J, Pearce K, Palmer C, Todd JK, Parker SK. Handshake stewardship: a Highly effective rounding-based antimicrobial optimization service. Pediatr Infect Dis J. 2016:35(10):1104-10.

31. Siegfried J, Merchan C, Scipione MR, Papadopoulos J, Dabestani A, Dubrovskaya Y. Role of postgraduate year 2 pharmacy residents in providing weekend antimicrobial stewardship coverage in an academic medical center. Am J Health Syst Pharm. 2017;74(6):417-23.

32. Nguyen-Ha PT, Howrie D, Crowley K, Vetterly CG, McGhee W, Berry D, et al. A quality assessment of a collaborative model of a pediatric antimicrobial stewardship program. Pediatrics. 2016;137(5):e20150316.

33. Guarascio AJ, Slain D, McKnight R, Petros K, Parker J, Wilson A, et al. A matched-control evaluation of an antifungal bundle in the intensive care unit at a university teaching hospital. Int J Clin Pharm. 2013;35(1):145-8.

34. Di Pentima MC, Chan S, Hossain J. Benefits of a pediatric antimicrobial stewardship program at a children's hospital. Pediatrics. 2011;128(6):1062-70.

35. Cappelletty D, Jacobs D. Evaluating the impact of a pharmacist's absence from an antimicrobial stewardship team. Am J Health Syst Pharm. 2013;70(12):1065-9.

36. Sutepvarnon A, Apisarnthanarak A, Camins B, Mondy K, Fraser VJ. Inappropriate use of antifungal medications in a tertiary care center in Thailand: a prospective study. Infect Control Hosp Epidemiol. 2008;29(4):370-3.

37. Karanika S, Paudel S, Grigoras C, Kalbasi A, Mylonakis E. Systematic review and meta-analysis of clinical and economic outcomes from the implementation of hospital-based antimicrobial stewardship programs. Antimicrob Agents Chemother. 2016;60(8):4840-52.

38. Apisarnthanarak A, Yatrasert A, Mundy LM, Thammasat University Antimicrobial Stewardship T. Impact of education and an antifungal stewardship program for candidiasis at a Thai tertiary care center. Infect Control Hosp Epidemiol. 2010;31(7):722-7.

\section{Publisher's Note}

Springer Nature remains neutral with regard to jurisdictional claims in published maps and institutional affiliations. 